Kuwada et al.

\title{
Pro-chemotherapeutic effects of antibody against extracellular domain of claudin-4 in
}

\section{bladder cancer}

Masaomi Kuwada ${ }^{1,2)}$, Yoshitomo Chihara ${ }^{2)}$, Yi Luo ${ }^{1)}$, Xiangru Li ${ }^{3)}$, Yukiko Nishiguchi ${ }^{1)}$, Rina Fujiwara $^{1)}$, Takamistu Sasaki ${ }^{4)}$, Kiyomu Fujii ${ }^{1)}$, Hitoshi Ohmori ${ }^{1)}$, Kiyohide Fujimoto ${ }^{2)}$, Masuo Kondoh ${ }^{3)}$, Hiroki Kuniyasu ${ }^{1)}$

${ }^{1)}$ Department of Molecular Pathology and ${ }^{2)}$ Department of Urology, Nara Medical University, Nara 634-8521, Japan

3) Laboratory of Bio-Functional Molecular Chemistry, Graduate School of Pharmaceutical Sciences, Osaka University, Osaka 565-0871, Japan

4) Department of Gastrointestinal Surgery, Fukuoka University School of Medicine 7-45-1, Japan

Running title: Claudin-4 targeting in bladder cancer

Key words: claudin, tight junction

Corresponding author: Hiroki Kuniyasu, at Department of Molecular Pathology, Nara

Medical University, 840 Shijo-cho, Kashihara, Nara, 634-8521, Japan. Tel: +81-744-22-3051,

FAX: +81-744-25-7308, e-mail: cooninh@zb4.so-net.ne.jp

Word count: 5172 words

Total number of figures and tables: 5 figures and 1 table 
Kuwada et al.

\begin{abstract}
Bladder cancer displays an aggressive phenotype in the muscle-invasive phase, and is associated with a high mortality rate. Therefore, novel molecular therapeutic targets are needed to improve patient survival. A monoclonal antibody against the extracellular domain of the claudin-4 (CLDN4) tight junction protein was established by immunizing rats with a plasmid vector encoding human CLDN4. A hybridoma clone, producing a rat monoclonal antibody recognizing CLDN4 (clone 4D3), was obtained. Immunohistochemistry by using the 4D3 antibody showed that CLDN4 expression was associated with local invasion, nodal metastasis, distant metastasis, and advanced stage in 86 cases of bladder cancer. The 4D3 antibody inhibited growth, invasion, and survival, associated with abrogation of the intratumoral microenvironment; lowered concentrations of epidermal growth factor and vascular endothelial growth factor were found in 3-dimentional cultures of T24 and RT4 cells. In combination with cisplatin therapy, 4D3 enhanced cisplatin cytotoxicity by increasing cellular permeability, leading to increased intracellular cisplatin concentrations. In mouse models of subcutaneous tumors and lung metastasis, 4D3 enhanced tumor growth inhibition, alone and with concurrent cisplatin treatment. The anti-tumor activity of the newly established 4D3 antibody suggests that it may be a powerful tool in CLDN4-targeting therapy, and in combination with chemotherapy. (197 words)
\end{abstract}

Abbreviation: MIUC; muscle invasive urothelial cancers, CLDN; Claudin, SPR; surface plasmon resonance, CDDP; Cisplatinum, EGFR; epidermal growth factor receptor, ERK; extracellular signal-regulated kinase, EGF; epidermal growth factor, VEGF; vascular endothelial growth factor, ADCC; antibody-dependent cellular cytotoxicity, CPE; Clostridium perfringens enterotoxin, C-CPE; C-terminal domain of CPE, ICG; indocyanin green, FITC; fluorescein isothiocyanate 
Kuwada et al.

\section{Introduction}

Bladder cancer was estimated to account for 15,000 deaths in the USA in 2013 [1], and accounted for 7,299 deaths in Japan in 2012 [2]. Approximately $80 \%$ of newly diagnosed bladder cancers are non-muscle-invasive urothelial tumors, which are treated with transurethral resection. Tumor recurrence and progression to invasive disease occurs in $70 \%$ and $15 \%$ of treated cases, respectively [3]. In contrast, muscle-invasive urothelial cancers (MIUC) display more aggressive behavior, with a 50\% mortality rate from disseminated disease [4]. Radical cystectomy is the gold-standard treatment for MIUC [5]. Some adjuvant chemotherapy trials have been carried out; a combination platinum-based regimen was associated with a 5-year survival advantage of 5-8\% over local therapy alone [6]. However, more effective chemotherapy regimens are needed to improve the overall prognosis for bladder cancer patients.

Claudins (CLDN) are protein components of tight junctions, which, together with adherens junctions and desmosomes, functionally seal adjacent cells together [7]. Twenty-seven different, but closely related, CLDN family members have been identified [8], all of which are thought to vary in expression, depending on location and cell type [9]. Tight junctions play a pivotal role in the maintenance of cell polarity by prevention of lateral diffusion of membrane proteins containing signaling molecules and lipids, which maintains the differential composition of the apical and basolateral domains [10], and subsequently, epithelial permeability and polarization [8]. Therefore, tight junctions are thought to be involved in the regulation of proliferation, differentiation, and other cellular functions.

CLDN4 is commonly expressed in epithelial tissues and solid tumors. Overexpression of CLDN4 is found in several types of epithelial malignancies, including pancreatic and ovarian cancer $[11 ; 12 ; 13 ; 14 ; 15 ; 16]$. CLDN4 expression is associated with malignant phenotypes, including metastasis, in cancer cells $[9 ; 17 ; 18]$. Ovarian cancer cells expressing CLDN4 show increased invasion, motility, and survival [19], and CLDN4 
Kuwada et al.

overexpression has been observed in advanced ovarian cancer [12]. CLDN4 expression has also been associated with a more invasive phenotype in pancreatic intraductal papillary mucinous neoplasms [16].

CLDN4 is thought to be a relevant molecular marker for diagnosis and treatment of epithelial cancers $[17 ; 18]$, and also a promising therapeutic target for drug development [20; 21]. In the present study, we established a monoclonal antibody (mAb) specific to the extracellular domain of CLDN4, to target CLDN4-expressing cells. The anti-tumor characteristics of the mAb were examined in bladder cancer models. 
Kuwada et al.

\section{Materials and Methods}

\subsection{Cells and reagents}

T24 and RT4 human bladder cancer cell lines were purchased from the American Type Culture Collection (ATCC; Manassas, VA, USA). Human CLDN (hCLDN)-expressing HT1080 cells were cultured in Dulbecco's modified Eagle's medium (DMEM), supplemented with $10 \%$ fetal bovine serum (FBS). Mouse CLDN (mCLDN)-expressing L cells, kindly provided by Dr. S. Tsukita (Kyoto University, Japan), were cultured in modified Eagle's medium (MEM) containing 10\% FBS.

\subsection{Cell growth and apoptosis}

Cell growth was assessed using a tetrazolium (MTT) dye assay, as previously described [22]. Apoptosis was assessed by examination of 2000 cells, stained with Hoechst 33342 dye (Life Technologies, Carlsbad, CA, USA), using a fluorescent microscope.

\subsection{Chamber invasion assay}

A modified Boyden chamber assay was performed to examine the in vitro invasion of colon cancer cells [23]. Following incubation at $37^{\circ} \mathrm{C}$ for 24 hours, the filters were carefully removed from the inserts, stained with hematoxylin for $10 \mathrm{~min}$, and mounted on microscopic slides. The number of stained cells in each insert was counted at 100x magnification. Invasion activity was quantified by calculating the average number of cells per insert well. These experiments were performed in triplicate.

\subsection{Reagents}

CM5 sensor chips, amine-coupling reagents (N-ethyl-N0-[3dimethylaminopropyl]-carbodiimide $\quad[$ EDC $], \quad \mathrm{N}$-hydroxysuccinimide $\quad[\mathrm{NHS}]$, and ethanolamine-HCl), and HBS-EP+ (10 mM HEPES, pH 7.4, $150 \mathrm{mM} \mathrm{NaCl,} 3 \mathrm{mM}$ EDTA, 
Kuwada et al.

and $0.05 \%$ surfactant P20) were obtained from GE Healthcare (Buckinghamshire, UK). Cisplatin (CDDP) was purchased from Novaplus (Bedford, OH, USA). All reagents used were of research grade.

\subsection{Animals}

Four-weeks-old BALB/c Slc-nu/nu mice and BALB/cCrSlc mouse were purchased from SLC Japan (Shizuoka, Japan). The mice were maintained according to the institutional guidelines approved by the Committee for Animal Experimentation of Nara Medical University, in accordance with the current regulations and standards of the Ministry of Health, Labor, and Welfare. Their sex and age were designated at each experimental method.

\subsection{Production of anti-human CLDN4 monoclonal antibody}

Wister rats were immunized with a eukaryotic expression vector encoding the full-length $\mathrm{h} C L D N 4$ cDNA, and the production of anti-hCLDN4 mAb in the serum was assessed by using fluorescence-activated cell sorter (FACS) analysis using hCDLN4-expressing HT1080 cells. B cells were isolated from rats with an increased serum titer of anti-hCLDN4 antibody, and the cells were fused with myeloma cells (P3U1), resulting in the production of hybridoma cells. Anti-hCLDN4 mAb-producing hybridoma cells were selected by using FACS, by their ability to bind to transient hCLDN4-expressing 293T cells and not to unexpressing 293T cells, using fluorescein-labeled goat anti-rat IgG $(\mathrm{H}+\mathrm{L})$ (Kirkegaard and Perry Laboratories, Gaithersburg, MD, USA) as a secondary antibody, resulting in the isolation of a rat anti-hCLDN4 mAb (clone 4D3). The immunoglobulin class was determined using a rat immunoglobulin isotyping enzyme-linked immunosorbant assay (ELISA) kit (BD Biosciences, San Jose, CA, USA).

\subsection{Preparation of hCLDN4 proteins}


Kuwada et al.

Recombinant hCLDN4 protein was prepared by using Sf9 cells infected with recombinant baculovirus, as previously reported [24]. Briefly, the C-terminal his-tagged hCLDN4 cDNA fragment was cloned into pFastBac1, and recombinant baculovirus was generated by using a Bac-to-Bac baculovirus expression system (Life Technologies). Sf9 cells were infected with the recombinant baculovirus. After $52-56 \mathrm{~h}$ of infection, the cells were harvested by centrifugation. The cells were washed with phosphate-buffered saline (PBS) and resuspended in $10 \mathrm{mM}$ Hepes buffer $(\mathrm{pH} 7.4,120 \mathrm{mM} \mathrm{NaCl})$ with protease inhibitor tablets (Complete Mini, EDTA-free) (Roche Applied Science, Indianapolis, IN, USA), $1 \mathrm{mM}$ phenylmethylsulfonyl fluoride, and 20 units/mL DNase I. The cells were lysed by the addition of $2 \%$ n-dodecyl-b-D-maltoside (DDM) and subsequently centrifuged. The resultant supernatant was applied to HisTrapTM HP (GE Healthcare), and hCLDN4 was eluted using imidazole. The solvent containing hCLDN4 was exchanged for PBS containing 0.2\% DDM, by gel filtration with a HiTrap Desalting column (GE Healthcare). Purification of CLDN4 was confirmed by sodium dodecyl sulfate polyacrylamide gel-electrophoresis (SDS-PAGE), followed by staining with coomassie brilliant blue (CBB).

\subsection{Surface plasmon resonance analysis}

Surface plasmon resonance (SPR) measurements were performed using a Biacore T200 instrument (GE healthcare). Amine-coupling chemistry was used to immobilize anti-rat mAb on a CM5 sensor chip surface in a Biacore TM200, and equilibrated with HBS-EP+. The carboxymethyl surface of the CM5 chip was activated with a 1:1 ratio of 0.4 M EDC and 0.1 M NHS. Anti-rat mAb was diluted to $20 \mu \mathrm{g} / \mathrm{mL}$ in $10 \mathrm{mM}$ acetate $(\mathrm{pH} 4.5)$ and applied to the chip surface. Excess activated groups were blocked by using $1 \mathrm{M}$ ethanolamine ( $\mathrm{pH}$ 8.5). Approximately $5000 \mathrm{RU}$ of anti-rat mAb were immobilized. The culture supernatant of 4D3-producing hybridoma cells was then applied to the chip surface for 2 min. hCLDN4 protein was serially diluted $(10,100,200,300$ and $500 \mathrm{nM})$ in HBS-EP+. Within a single 
Kuwada et al.

binding cycle, hCLDN4 protein was applied sequentially, in order of increasing concentration, over both the ligand and the reference surfaces. The reference surface, an unmodified flowcell, was used to correct for systematic noise and instrumental drift. The sensorgrams were globally fitted by using a 1:1 binding model to determine association rate (ka), dissociation rate $(\mathrm{kd})$, and equilibrium dissociation constant (KD) values, using Biacore T200 Evaluation Software (GE Healthcare).

\subsection{Purification of rat anti-human CLDN4 monoclonal antibody}

Eight-week-old female BALB/c Slc-nu/nu mice (SLC) were intraperitoneally administered with pristane as an immunological adjuvant, and $1 \times 10^{7} 4 \mathrm{D} 3$-producing hybridoma cells. Ascites was collected, and the mAb (4D3) was purified by using Ab-Capcher ExTra (ProteNova, Kagawa, Japan). Eluted $\mathrm{mAb}$ was dialyzed into PBS, and the protein concentration was determined by measuring absorbance at $280 \mathrm{~nm}$. The purity of the mAb was confirmed by SDS-PAGE, followed by staining with CBB.

\subsection{CLDN specificity analysis}

To analyze specific binding of 4D3 to CLDN-expressing cells, hCLDN-expressing HT1080 cells and mCLDN4-expressing L cells were detached and incubated with $5 \mu \mathrm{g} / \mathrm{mL}$ of 4D3, followed by treatment with secondary fluorescein-conjugated goat anti-rat $\operatorname{IgG}(\mathrm{H}+\mathrm{L})$ (Kirkegaard and Perry Laboratories). The mAb-bound cells were analyzed by using FACSCalibur and CellQuestPro software (BD Biosciences).

\subsection{Patients}

Eighty-six cases of bladder cancer that had been treated with transurethral resection or total cystectomy in Nara Medical University Hospital were randomly selected. Basic patient information is summarized in Table 1. As written informed consent was not obtained, any 
Kuwada et al.

identifying information was removed from the samples prior to analysis, to ensure strict privacy protection (unlinkable anonymization). All procedures were performed in accordance with the Ethical Guidelines for Human Genome/Gene Research enacted by the Japanese Government, which was approved by the Ethics Committee of Nara Medical University (Approval Number 937).

\subsection{Immunohistochemistry}

Consecutive 4- $\mu \mathrm{m}$ sections were immunohistochemically stained using the immunoperoxidase technique described previously [25]. 4D3 was used at a concentration of $0.2 \mu \mathrm{g} / \mathrm{mL}$. Secondary antibodies (Medical and Biological Laboratories, Nagoya, Japan) were used at a concentration of $0.2 \mu \mathrm{g} / \mathrm{mL}$. Tissue sections were color-developed with diamine benzidine hydrochloride (DAKO, Glastrup, Denmark), and counterstained with Meyer's hematoxylin (Sigma Chemical Co., St. Louis, MO, USA). We count the cells with the immunoreaction at the cytoplasmic membrane. Staining strength was scored from 0 to 3 (a score of 1 was used to describe the expression level in normal urothelium). The staining index was calculated as the staining strength score multiplied by the staining area $(\%)$, and the resulting scores were defined as follows: none (index, 0), weak (index, 1-100), medium (index, 101-200), and high (index, 201-300). For a negative control, unimmunized rat IgG (Santa-Cruz Biotechnology, Santa-Cruz, CA, USA) was used as primary antibody.

\subsection{Reverse transcription-polymerase chain reaction}

Reverse transcription-polymerase chain reaction (RT-PCR), using $0.5 \mu \mathrm{g}$ total RNA extracted using an RNeasy kit (Qiagen, Germanytown, MD, USA), was used to assess human CLDN4 mRNA expression. The primer sets for human CLDN4 amplification were as follows: forward 5'-CTC CAT GGG GCT ACA GGT AA-3' and reverse 5'-AGC AGC GAG TCG TAC ACC TT-3' (NCBI Reference Sequence: NM_001305.4; synthesized by Sigma Genosys, Ishikari, 
Kuwada et al.

Japan). PCR products were electrophoresed in a 2\% agarose gel and stained with ethidium bromide. $\beta$-actin $(A C T B)$ mRNA was also amplified for use as an internal control (GenBank Accession No. NM_001101).

\subsection{Quantitative reverse transcription-polymerase chain reaction}

Extraction of total RNA was carried out using an RNeasy Mini Kit (Qiagen), and total RNA

$(1 \mu \mathrm{g})$ was synthesized using the ReverTra Ace- $\alpha$-RT Kit (Toyobo, Osaka, Japan).

Quantitative reverse transcription-polymerase chain reaction (qRT-PCR) was performed by using StepOne Real-Time PCR System with Fast SYBR ${ }^{\circledR}$ Green Master Mix (Applied Biosystems, Life Technologies, Carlsbad, CA, USA), and a relative standard curve quantification method for used for analysis [26]. PCR conditions were used according to the manufacturer's instructions. ACTB mRNA was amplified as an internal control (GenBank accession No. NM 001101). Each amplification reaction was evaluated by using a melting curve analysis. PCR products were visualized using agarose gel electrophoresis with ethidium bromide staining.

\subsection{Immunoblot analysis}

Whole-cell lysates were prepared as previously described [23]. Lysates (50 $\mu \mathrm{g}$ ) were subjected to immunoblot analysis using SDS-PAGE (12.5\%), followed by electrotransfer onto nitrocellulose filters. The filters were incubated with primary antibodies, followed by peroxidase-conjugated IgG antibodies (MBL, Nagoya, Japan). Anti-tubulin antibody was used to assess the levels of protein loaded per lane (Oncogene Research Products, Cambridge, MA, USA). The immune complex was visualized using an ECL Western-blot detection system (Amersham, Aylesbury, UK). Antibody for CLDN1 (clone 2C1) was established by us [27]. Antibodies for epidermal growth factor receptor (EGFR; Cell Signaling Technology, Beverly, MA, USA), phosphorylated EGFR (pTyr992, Cell Signaling Technology), extracellular 
Kuwada et al.

signal-regulated kinase 1/2 (ERK1/2; Santa-Cruz) and phosphorylated ERK1/2 (Santa-Cruz) matrix metalloproteinase 9 (MMP9, Novocastra Laboratories, Newcastle upon Tyne, UK), CLDN2, 3, 5, 6, 7, 8, and 9 (rabbit polyclonal, Biorbyt, Cambridge, UK) were used as primary antibodies.

\subsection{Immunoprecipitation}

For immunoprecipitation, the lysates were pre-cleaned in lysis buffer with protein $A / G$ agarose (Santa-Cruz) for 1 hour at $4^{\circ} \mathrm{C}$ and subsequently centrifuged. The supernatants were incubated with anti-CLDN4 antibody (4D3) or anti-CLDN1 antibody (2C1) and protein A/G agarose for 16 hours at $4^{\circ} \mathrm{C}$. Precipitates were collected by centrifugation and washed 5 times with lysis buffer for SDS-PAGE. The precipitants were detected by antbodies for CLDN4 (4D3), CLDN1 (2C1), CLDN3 and CLDN7 (Origene, Rockville, MD, USA). For loading control, five $\mu 1$ of each preimmunoprecipitated sample (lysate diluted with buffer) was slot-blotted onto nitrocellulose membrane and stained with Coomassie Blue.

\subsection{Short interfering $R N A$}

FlexiTube short interfering RNA (siRNA) for CLDN4 was purchased from Santa-Cruz. AllStars Negative Control siRNA was used as a control (Qiagen). Cells were transfected with $50 \mathrm{nM}$ siRNA using Lipofectamine 2000 (Invitrogen), according to the manufacturer's instructions.

\subsection{Intracellular platinum}

$\mathrm{T} 24$ cells $\left(1 \times 10^{10}\right)$ were digested with proteinase $\mathrm{K}$ for $2 \mathrm{~h}$ at $45^{\circ} \mathrm{C}$, followed by treatment with $65 \%$ nitric acid overnight at $80^{\circ} \mathrm{C}$. The suspension was analyzed using a flameless atomic absorption spectrometer (AAS) to measure the platinum concentration $(\lambda=265.9 \mathrm{~nm})$, using an AAS platinum standard (Sigma-Aldrich, St Louis, MO, USA). 
Kuwada et al.

\subsection{In vitro permeability assay}

To carry out multiple cell layer insert assays, T24 cells $\left(1 \times 10^{4}\right)$ were seeded on the bottom of uncoated insert wells (pore size, $3 \mu \mathrm{m}$; diameter, $5 \mathrm{~mm}$; BD biosciences), and the culture medium was changed to DMEM with $2 \%$ bovine serum albumin (BSA; Sigma-Aldrich). peroxidase-conjugated mouse IgG (IgG-PO; $0.5 \mu \mathrm{g} / \mathrm{mL}$; DAKO) was added to the insert chambers. After cells were treated with 4D3 antibody for $6 \mathrm{~h}$, media from the inserts and lower chambers were collected into a 96-well ELISA plate, which was color-developed with the addition of PO $(0.5 \mu \mathrm{g} / \mathrm{mL})$ or TMB $(10 \% \mathrm{v} / \mathrm{v})$. The reaction was stopped by the addition of $2 \mathrm{~N} \mathrm{H}_{2} \mathrm{SO}_{4}$, and the optical density at $450 \mathrm{~nm}(\mathrm{OD} 450)$ was measured.

An InVitro Vascular Permeability Kit (Trevigen Inc. Gaithersburg, MD) was used to determine permeation of fluorescein isothiocyanate (FITC)-dextran in a monolayer of T24 or RT4 cells, according to the maufacturer's instruction. Cytochalasin B $(1 \mu \mathrm{g} / \mathrm{mL})$ was used as a positive control [28].

To carry out a multiple cell layer microcarrier assay, $10 \mathrm{mg}$ of microcarrier (Corning Inc., Corning, NY, USA) was incubated with T24 cells $\left(1 \times 10^{4}\right)$. After $48 \mathrm{~h}$, microcarriers were collected by using filter paper and subsequent centrifugation at $5,000 \mathrm{xg}$, for $5 \mathrm{~min}$, at $4^{\circ} \mathrm{C}$. The supernatant was used for subsequent ELISA.

\subsection{Enzyme-linked immunosorbant assay}

ELISA kits were used to measure protein concentrations of epidermal growth factor (EGF) (R\&D Systems Inc., Minneapolis, MN, USA), and vascular endothelial growth factor (VEGF) (Abcam, Cambridge, UK), according to manufacturers' instructions.

\subsection{Animal tumor models}

To establish a subcutaneous tumor model, T24 cancer cells $\left(1 \times 10^{7}\right)$ were inoculated into the 
Kuwada et al.

scapular subcutaneous tissues of nude mice. For the lung metastasis model, T24 cancer cells $\left(1 \times 10^{6}\right)$ were inoculated into the caudal vein. Mice lungs were observed for 4 weeks following inoculation. The lungs were slices by $2 \mathrm{~mm}$-thickness and the metastatic foci were counted under observation using a stereomicroscope (Nikon, Tokyo, Japan). CDDP and/or 4D3 were administrated into the peritoneal cavity.

For the subcutaneous model, CDDP (3mg/kg body weight, diluted with saline) and/or 4D3 $(1 \mathrm{mg} / \mathrm{kg}$ body weight, diluted with saline) were injected into the peritoneal cavity simultaneously on Day 1, 3, and 7. For the lung cancer model, CDDP (3mg/kg body weight) and/or 4D3 (1 $\mathrm{mg} / \mathrm{kg}$ body weight) were injected into the peritoneal cavity simultaneously on Day $1,8,15$, and 22. In both models, each group contained 5 mice.

\subsection{Antibody accumulation in the tumor}

Accumulation of 4D3 was examined by whole mouse imaging and frozen section of the tumor tissue. T24 cancer cells $\left(1 \times 10^{7}\right)$ were inoculated into the scapular subcutaneous tissues of nude mice. For whole mouse imaging, indocyanin green (ICG)-labeled 4D3 (1 mg/kg body weight, labeled by ICG-labeling kit [Dojindo, Kumamoto, Japan]) was injected i.p. on Day 10 of the subcutaneous tumor [29]. A mouse was examined by Clairvivo OPT in vivo imager (Shimazu, Kyoto, Japan) under anesthesia [29]. For examination of intratumoral localization, FITC-labeled 4D3 (1 mg/kg body weight, labeled by FITC Antibody Labeling Kit [Pierce, Rockford, IL]) was injected i.p. on Day 14 of the subcutaneous tumor. At 6 hrs after injection tumor was excised to make frozen section and observed by a fluorescent microscope (Leica, Buffalo Grove, IL).

\subsection{Statistical analysis}

Statistical significance was calculated by using two-tailed Fisher's exact, Chi-square, and unpaired Mann-Whitney tests by using InStat software (Graphpad, Los Angeles, CA, USA). 
Kuwada et al.

Survival curves were calculated by using a Kaplan-Meier model (Statview 4.5, Abacus Concepts, Inc., Berkeley, CA, USA). Differences in survival were calculated by using the Cox proportional hazard model (Statview 4.5). Statistical significance was defined as a two-sided $p$-value of $<0.05$. 
Kuwada et al.

\section{Results}

\subsection{Creation of an anti-hCLDN4 monoclonal antibody}

We generated an anti-CLDN4 mAb (4D3) by genetic immunization using a full-length CLDN4 cDNA expression vector. Analysis of antibody isotypes revealed that the 4D3 isotype was IgG2b. SPR analysis of 4D3 showed the following binding kinetics: ka value, $1.99 \times 10^{4}$ 1/Ms; kd value, $8.27 \times 10^{-4} 1 / \mathrm{s}$, and $\mathrm{KD}$ value, $41.5 \mathrm{nM}$. 4D3 bound to hCLDN4-expressing cells, but not to hCLDN1, 2, 3, 5, 6, 7, 9, or mCLDN4-expressing cells (Fig. 1A).

To confirm the specificity of the 4D3 antibody, an immunoprecipitation was performed (Fig. 1B). Lysate of T24 cells were immunoprecipitaed with 4D3 or anti-CLDN1 antibody (2C1). CLDN4 was detected by 4D3 in the 4D3-precipitant alone. CLDN4 knockdown abrogated CLDN4 precipitation. Moreover, CLDN1, 3, or 7 was not detected in 4D3-precipitants by their specific antibodies. Therefore, 4D3 is a specific mAb to hCLDN4.

We also examined the endogenous protein expression levels of hCLDN1, 2, 3, 4, 5, 6, 7, 8, and 9 in HT1080 cells, which were treated with control siRNA or CLDN4 siRNA (Fig. 1C). The constitutive expression of hCLDN4 was undetectable in HT1080 cells. HT1080 cells transfected with human CLDN4 gene (HT1080-hCLDN4 cells), were treated with control siRNA or CLDN4 siRNA. CLDN4 expression was disappeared in CLDN4-knockdowned cells, whereas the expressions of hCLDN1, 2, 3, 5, 6, 7, 8, and 9 were not affected by CLDN4 knockdown.

\subsection{Expression of CLDN4 in human bladder cancer detected by the 4D3 monoclonal antibody}

4D3 detects the expression of CLDN4 by binding to its extracellular domain, in contrast to other available anti-CLDN4 antibodies. CLDN4 was detected at intercellular borders in non-cancerous urothelium and low-grade/non-invasive cancer (Fig. 2A-B), whereas it was overexpressed and located at the cytoplasmic membrane and in the cytoplasm in high-grade, 
Kuwada et al.

invasive, and metastasizing cancers (Fig. 2C-D). In contrast, unimmunized rat IgG showed no signals (Fig. 2E). Results from the examination of 86 bladder cancer tumors are shown in Table 1. CLDN4 expression was significantly associated with local invasion (pT, $P=0.0123$ ), nodal metastasis (pN, $P=0.0059)$, distant metastasis (pM, $P=0.0341)$, and pathological stage $(P=0.0011)$, but not with histological grade. Especially, the two cases of pM1 showed metastasis to the bone and the lung. The mean staining index (strength $\mathrm{x}$ area) of CLDN4 in the two cases $(275 \pm 7)$ expressions were significantly higher than that in the pM0 cases (146 $\pm 69)(\mathrm{P}=0.0101)$.

\subsection{Effect of $4 D 3$ on bladder cancer cells}

The human bladder cancer cell lines, RT4 (low-grade cancer derived) and T24 (high-grade cancer derived) expressed CLDN4 mRNA and protein (Fig 3A). Cell growth and invasion of RT4 and T24 cells were modestly inhibited by 4D3 in a dose-dependent manner, whereas apoptosis was increased by 4D3 in a dose-dependent manner (Fig. 3B-D).

Multicarrier assays showed that intracarrier EGF concentration was significantly reduced by 4D3 treatment (Fig. 3E). Subsequent phosphorylation of EGFR and ERK1/2 was also decreased by 4D3 treatment (Fig. 3F). In the same assay, intracarrier VEGF concentration was significantly reduced by 4D3 treatment (Fig. 3G). 4D3-treated cells showed lower mRNA expression of $V E G F$ and $H I F-1 \alpha$, which suggests an improvement in hypoxic conditions (Fig. 3H).

\subsection{Effects of $4 D 3$ on in vitro bladder cancer cells concurrently treated with cisplatin}

CDDP is the most common chemotherapeutic agent used in bladder cancer treatment, and we examined the effects of concurrent 4D3 and CDDP treatment on bladder cancer cells (Fig. 4). CDDP caused growth inhibition of T24 and RT4 cells in a dose-dependent manner. Treatment with 4D3 enhanced the inhibition of cell growth caused by CDDP, in both cell lines (Fig. 4A- 
Kuwada et al.

B). Intracellular platinum concentration was significantly increased in cells treated with 4D3 compared to those without 4D3 treatment (Fig. 4C-D).

Using a multiple cell layer permeability assay, peroxidase-labeled IgG were added to the upper chamber and permeated through the T24 cell layer into the lower chamber, in a 4D3 dose-dependent manner (Fig. 4E). In contrast, a control IgG did not affect the permeability. Using a monolayer permeability assay, FITC-dextran was shielded by T24 or RT4 cell layers. 4D3 treatment inhibited shielding at the same levels as non-cell or cytochalasine B treatment (Fig. 4F).

\subsection{Effect of 4D3 on in vivo bladder cancer cells concurrently treated with cisplatin}

We next examined the effect of concurrent 4D3 and CDDP treatment on subcutaneous tumors in nude mice (Fig. 5A). Administration of 4D3 and CDDP three times in week 1 inhibited tumor growth of subcutaneous T24 cell tumors. In tumors, mice treated with 4D3 alone showed $23 \%$ inhibition of tumor growth compared to those with untreated tumors. Mice treated with CDDP or CDDP combined with 4D3 (CDDP+4D3) showed tumor growth inhibition of $52 \%$ and $70 \%$, respectively. In the lung metastasis model, treatment with 4D3, CDDP, or CDDP+4D3 decreased the number of lung metastasis by $29 \%, 50 \%$, and $74 \%$, respectively, at week 4 (Fig. 5B).

The localization of the administrated 4D3 was examined by detection of ICG-labeled 4D3 in whole mouse imaging (Fig. 5C) and of FITC-labeled 4D3 in the tumor tissue by fluorescent microscopy in frozen section (Fig. 5D). 4D3 accumulation at the subcutaneous tumor was found by whole mouse imaging. 4D3 immunoreactivity was observed on the cell-cell boundary, which is compatible to the tight junction.

To confirm the enhancement of permeability of cancer tissue, FITC-dextran was administrated for subsequent detection in the tumors (Fig. 5E-F). FITC-dextran infiltrated the intracellular spaces more prominently in 4D3-treated mice than in IgG-treated control mice 
Kuwada et al.

(Fig. 5E). The permeated areas were also significantly larger in 4D3-treated mice than that in IgG-treated control mice (Fig. 5F).

The effect of the treatment was examined by analyzing survival in mice (Fig. 5F). In the T24 subcutaneous tumor model, survival was significantly improved by CDDP or CDDP+4D3 treatment (Fig. 5G). The survival rates at day 28 were $0 \%, 0 \%, 33 \%$ and $50 \%$ in the untreated mice, and 4D3, CDDP, and CDDP+4D3-treated mice, respectively. The mice treated with $\mathrm{CDDP}+4 \mathrm{D} 3$ showed significantly improved survival than that in the untreated $\operatorname{mice}(P<0.01)$. 
Kuwada et al.

\section{Discussion}

CLDN is an essential protein in maintaining cell polarity and molecular trafficking, via tight junctions. In this study, we established an antibody to the extracellular domain of CLDN4, which is only the second antibody of this kind [30]. When CLDN expression is increased, the barrier function of cancerous epithelia changes, and it often displays a disorganized arrangement of tight junction strands, with increased permeability to paracellular markers [31]. Dysregulation of these functions contributes to initiation and progression of cancer [8]. Altered CLDN expression has been reported in many types of epithelial cancers in a stageand tumor-specific manner [8; 31], and the present study shows overexpression of CLDN4 in bladder cancer.

Our data demonstrates that the 4D3 anti-CLDN4 antibody inhibited growth, invasion, and survival of cancer cells in the condition of in vitro or of the mouse tumor models. Considering that anti-CLDN4 antibody, 4D3 recognized the extracellu.ar loop 1 and 2, binding of 4D3 to CLDN4 is thought to block homophilic trans-interaction, which forms gap between 2 strands of tight junctions. The gap abrogates barrier- and fence-functions of tumor tissues $[32 ; 33]$. We then evaluated the loss of tight junctions in cancer tissue using two different methods. First, we examined the confinement of EGF, a cancer-stimulating growth factor, by tight junctions by using microcarrier beads. Treatment with the 4D3 anti-CLDN4 antibody allowed leakage of EGF from the beads, which suppressed phosphorylation of EGFR and ERK1/2 and also expression of MMP9. Thus the tumor growth and invasion might be inhibited by $4 \mathrm{D} 3$ treatment. In contrast, CLDN4 maintains the internal environment of the tumor, which induces HIF-1 $\alpha$ and VEGF due to the hypoxic condition. CLDN4 thus affects the expression and secretion of these factors and also the signal pathway by an indirect manner.

Second, we examined the diffusion of anti-cancer molecules into the intercellular space treated with 4D3. Anti-CLDN4 antibody treatment increased permeability of multiple 
Kuwada et al.

cell layers allowing permeation of CDDP into tumor tissue, and subsequent intracellular accumulation. These effects inhibit cancer cells and increase the efficacy of anti-cancer drugs. It has been reported that an increase in vascular permeability leads to enhanced anti-cancer drug concentrations in tumor tissue [34], but as CLDN4 is not involved in endothelial tight junctions [35], we believe that the effect of the anti-CLDN4 antibody reflects the increased permeability of cancer cell tight junction. CLDN4 has also been reported to regulate sensitivity to CDDP by controlling the expression of copper and the CDDP influx transporter, CTR1 [36], but these modes of CDDP regulation by CLDN4 have not been examined in this study.

In some types of malignancies, an intrinsic decrease in CLDN4 expression has been associated with cancer cell discohesion, invasion, and metastasis [37; 38]. Knockdown of CLDN4 was shown to reduce tight junction formation, transepithelial resistance, and the paracellular flux of dextran, which enhances migration and invasion in in vitro assays, and increases lung metastasis [37]. In contrast, our data did not detect any effects of inhibition of CLDN4 by 4D3 in cancer cells with a metastatic phenotype. We also found that advanced cases of bladder cancer expressed CLDN4 at higher levels than early cases, suggesting that CLDN4 overexpression may be associated with tumor progression in bladder cancer, a different role than that associated with ovarian and colorectal cancer [37; 38]. Our data suggests that the role of CLDN4 in sustaining the internal microenvironment of the tumor might be advantageous for bladder cancer. Increased CLDN4 expression is epigenetically regulated by hypomethylation of CLDN4 in gastric cancer [39]. In contrast, during ovarian tumorigenesis, derepression of CLDN4 expression correlates with loss of trimethylated histone H3 lysine 27 and H4 lysine 20, in addition to DNA hypermethylation [40].

CLDN4 is a widely distributed molecule in various epithelial tissues. Then toxicity by the targeting of CLDN4 is an essential issue. Because 4D3 antibody recognizes only human CLDN4 but not CLDN4 of rodent or other animals, evaluation of the toxicity using 
Kuwada et al.

animals is not relevant. In literature, CLDN4 knockout mouse was reported [41]. The CLDN4-knockout mice show urothelial hyperplasia and lethal hydronephrosis; however, hetero-deficient mice do not show above anomaly. From the results, in the normal tissues, CLDN4 deficiency might result none or traceable dysfunction of tight junction. C.perfringens toxin binds to both CLDN4 and CLDN3, and it provides pore formation in the plasma membrane [42], which are causes of the strong cytotoxicity. For confirmation of safety of the antibody, evaluation by using primates models is thought to be good for preclinical examination.

Therapeutic targeting of CLDN4 has increasingly been proposed in the literature [43; 44; 45]. Several reports have recommended the use of bacterial toxins, such as Clostridium perfringens enterotoxin (CPE) and the $\mathrm{C}$-terminal domain of CPE (C-CPE) as CLDN4 binders [43; 44]. It has also been demonstrated that CLDN4 targeting is effective for treatment of cancer metastasis, by using C-CPE fused to a protein synthesis inhibitory factor [45]. We have also established bispecific antibodies to CLDN3 and 4, which show anti-tumor activity in in vivo and in vitro experiments [46]. The anti-tumor activity of the newly established 4D3 anti-CLDN4 antibody that we have demonstrated in this study, suggests that it might be effective in CLDN4-targeting therapy, alone and in combination with chemotherapy. 
Kuwada et al.

\section{Acknowledgements}

Health and Labour Sciences Research Grant from the Ministry of Health, Labour and Welfare of Japan (12855223); Japan Agency for Medical Research and Development (15653182); Grant-in-Aid for Scientific Research from the Ministry of Education, Culture, Sports, Science, and Technology of Japan (24390042); the Adaptable and Seamless Technology Transfer Program through Target-driven R\&D, Japan Science and Technology Agency (AS242Z01694Q and AS251Z00905Q); the Takeda Science Foundation; the Platform for

Drug Discovery, Informatics, and Structural Life Science of the Ministry of Education, Culture, Sports, Science, and Technology, Japan.

Conflict of interest statement

None 
Kuwada et al.

\section{References}

[1] R. Siegel, J. Ma, Z. Zou, A. Jemal, Cancer statistics, 2014. CA Cancer J Clin 64 (2014) 9-29.

[2] F. Wakao, H. Nishimoto, K. Kataonoda, H. Tsukuma, H. Mikami, (Eds.) Cancer Statistics in Japan, 2013, National Cancer Research Institute, Tokyo, 2013.

[3] K. Sugano, T. Kakizoe, Genetic alterations in bladder cancer and their clinical applications in molecular tumor staging. Nat Clin Pract Urol 3 (2006) 642-652.

[4] A.M. Aparicio, A.B. Elkhouiery, D.I. Quinn, The current and future application of adjuvant systemic chemotherapy in patients with bladder cancer following cystectomy. Urol Clin North Am 32 (2005) 217-230, vii.

[5] M.A. Ghoneim, H. Abol-Enein, Management of muscle-invasive bladder cancer: an update. Nat Clin Pract Urol 5 (2008) 501-508.

[6] J.C. Herring, A.M. Kamat, Treatment of muscle-invasive bladder cancer: progress and new challenges. Expert Rev Anticancer Ther 4 (2004) 1047-1056.

[7] S. Tsukita, M. Furuse, M. Itoh, Multifunctional strands in tight junctions. Nat Rev Mol Cell Biol 2 (2001) 285-293.

[8] K. Turksen, T.C. Troy, Junctions gone bad: claudins and loss of the barrier in cancer. Biochim Biophys Acta 1816 (2011) 73-79.

[9] A. Escudero-Esparza, W.G. Jiang, T.A. Martin, The Claudin family and its role in cancer and metastasis. Front Biosci (Landmark Ed) 16 (2011) 1069-1083.

[10] L.L. Mitic, J.M. Anderson, Molecular architecture of tight junctions. Annu Rev Physiol 60 (1998) 121-142.

[11] C.D. Hough, C.A. Sherman-Baust, E.S. Pizer, F.J. Montz, D.D. Im, N.B. Rosenshein, K.R. Cho, G.J. Riggins, P.J. Morin, Large-scale serial analysis of gene expression reveals genes differentially expressed in ovarian cancer. Cancer Res 60 (2000) $6281-6287$. 
[12] L.B. Rangel, R. Agarwal, T. D'Souza, E.S. Pizer, P.L. Alo, W.D. Lancaster, L. Gregoire, D.R. Schwartz, K.R. Cho, P.J. Morin, Tight junction proteins claudin-3 and claudin-4 are frequently overexpressed in ovarian cancer but not in ovarian cystadenomas. Clin Cancer Res 9 (2003) 2567-2575.

[13] S.L. Kominsky, M. Vali, D. Korz, T.G. Gabig, S.A. Weitzman, P. Argani, S. Sukumar, Clostridium perfringens enterotoxin elicits rapid and specific cytolysis of breast carcinoma cells mediated through tight junction proteins claudin 3 and 4. Am J Pathol 164 (2004) 1627-1633.

[14] H. Long, C.D. Crean, W.H. Lee, O.W. Cummings, T.G. Gabig, Expression of Clostridium perfringens enterotoxin receptors claudin-3 and claudin-4 in prostate cancer epithelium. Cancer Res 61 (2001) 7878-7881.

[15] P. Michl, M. Buchholz, M. Rolke, S. Kunsch, M. Lohr, B. McClane, S. Tsukita, G. Leder, G. Adler, T.M. Gress, Claudin-4: a new target for pancreatic cancer treatment using Clostridium perfringens enterotoxin. Gastroenterology 121 (2001) 678-684.

[16] N. Sato, N. Fukushima, A. Maitra, C.A. Iacobuzio-Donahue, N.T. van Heek, J.L. Cameron, C.J. Yeo, R.H. Hruban, M. Goggins, Gene expression profiling identifies genes associated with invasive intraductal papillary mucinous neoplasms of the pancreas. Am J Pathol 164 (2004) 903-914.

[17] P.J. Morin, Claudin proteins in human cancer: promising new targets for diagnosis and therapy. Cancer Res 65 (2005) 9603-9606.

[18] A. Neesse, H. Griesmann, T.M. Gress, P. Michl, Claudin-4 as therapeutic target in cancer. Arch Biochem Biophys 524 (2012) 64-70.

[19] R. Agarwal, T. D'Souza, P.J. Morin, Claudin-3 and claudin-4 expression in ovarian epithelial cells enhances invasion and is associated with increased matrix metalloproteinase-2 activity. Cancer Res 65 (2005) 7378-7385.

[20] A. Takahashi, M. Kondoh, H. Suzuki, K. Yagi, Claudin as a target for drug development. 
Kuwada et al.

Curr Med Chem 18 (2011) 1861-1865.

[21] K. Matsuhisa, M. Kondoh, H. Suzuki, K. Yagi, Comparison of mucosal absorption-enhancing activity between a claudin-3/-4 binder and a broadly specific claudin binder. Biochem Biophys Res Commun 423 (2012) 229-233.

[22] H. Kuniyasu, S. Yano, T. Sasaki, T. Sasahira, S. Sone, H. Ohmori, Colon cancer cell-derived high mobility group 1/amphoterin induces growth inhibition and apoptosis in macrophages. Am J Pathol 166 (2005) 751-760.

[23] H. Kuniyasu, N. Oue, A. Wakikawa, H. Shigeishi, N. Matsutani, K. Kuraoka, R. Ito, H. Yokozaki, W. Yasui, Expression of receptors for advanced glycation end-products (RAGE) is closely associated with the invasive and metastatic activity of gastric cancer. J Pathol 196 (2002) 163-170.

[24] H. Uchida, M. Kondoh, T. Hanada, A. Takahashi, T. Hamakubo, K. Yagi, A claudin-4 modulator enhances the mucosal absorption of a biologically active peptide. Biochem Pharmacol 79 (2010) 1437-1444.

[25] H. Kuniyasu, W. Yasui, H. Shinohara, S. Yano, L.M. Ellis, M.R. Wilson, C.D. Bucana, T. Rikita, E. Tahara, I.J. Fidler, Induction of angiogenesis by hyperplastic colonic mucosa adjacent to colon cancer. Am J Pathol 157 (2000) 1523-1535.

[26] K.J. Livak, T.D. Schmittgen, Analysis of relative gene expression data using real-time quantitative PCR and the 2(-Delta Delta C(T)) Method. Methods 25 (2001) 402-408.

[27] M. Fukasawa, S. Nagase, Y. Shirasago, M. Iida, M. Yamashita, K. Endo, K. Yagi, T. Suzuki, T. Wakita, K. Hanada, H. Kuniyasu, M. Kondoh, Monoclonal antibodies against extracellular domains of claudin-1 block hepatitis $\mathrm{C}$ virus infection in a mouse model. $\mathrm{J}$ Virol 89 (2015) 4866-4879.

[28] X.J. Liang, J.J. Yin, B. Taylor, S.M. Winkovitch, S.H. Garfield, D.W. Shen, M.M. Gottesman, A. Aszalos, Disruption of microfilaments by cytochalasin B decreases accumulation of cisplatin in human epidermal carcinoma and liver carcinoma cell lines. 
Kuwada et al.

Cancer Chemother Pharmacol 62 (2008) 977-984.

[29] M. Ogawa, C.A. Regino, J. Seidel, M.V. Green, W. Xi, M. Williams, N. Kosaka, P.L. Choyke, H. Kobayashi, Dual-modality molecular imaging using antibodies labeled with activatable fluorescence and a radionuclide for specific and quantitative targeted cancer detection. Bioconjug Chem 20 (2009) 2177-2184.

[30] M. Suzuki, M. Kato-Nakano, S. Kawamoto, A. Furuya, Y. Abe, H. Misaka, N. Kimoto, K. Nakamura, S. Ohta, H. Ando, Therapeutic antitumor efficacy of monoclonal antibody against Claudin-4 for pancreatic and ovarian cancers. Cancer Sci 100 (2009) 1623-1630.

[31] L. Ding, Z. Lu, Q. Lu, Y.H. Chen, The claudin family of proteins in human malignancy: a clinical perspective. Cancer Manag Res 5 (2013) 367-375.

[32] J.M. Anderson, C.M. Van Itallie, Physiology and function of the tight junction. Cold Spring Harb Perspect Biol 1 (2009) a002584.

[33] M. Furuse, K. Fujita, T. Hiiragi, K. Fujimoto, S. Tsukita, Claudin-1 and -2: novel integral membrane proteins localizing at tight junctions with no sequence similarity to occludin. J Cell Biol 141 (1998) 1539-1550.

[34] K. Greish, Enhanced permeability and retention of macromolecular drugs in solid tumors: a royal gate for targeted anticancer nanomedicines. J Drug Target 15 (2007) 457-464.

[35] C. Forster, Tight junctions and the modulation of barrier function in disease. Histochem Cell Biol 130 (2008) 55-70.

[36] X. Shang, X. Lin, G. Manorek, S.B. Howell, Claudin-3 and claudin-4 regulate sensitivity to cisplatin by controlling expression of the copper and cisplatin influx transporter CTR1. Mol Pharmacol 83 (2013) 85-94.

[37] X. Shang, X. Lin, E. Alvarez, G. Manorek, S.B. Howell, Tight junction proteins claudin-3 and claudin-4 control tumor growth and metastases. Neoplasia 14 (2012) 974-985. 
Kuwada et al.

[38] D. Suren, M. Yildirim, V. Kaya, A.S. Alikanoglu, N. Bulbuller, M. Yildiz, C. Sezer, Loss of tight junction proteins (Claudin 1, 4, and 7) correlates with aggressive behavior in colorectal carcinoma. Med Sci Monit 20 (2014) 1255-1262.

[39] M.J. Kwon, S.H. Kim, H.M. Jeong, H.S. Jung, S.S. Kim, J.E. Lee, M.C. Gye, O.C. Erkin, S.S. Koh, Y.L. Choi, C.K. Park, Y.K. Shin, Claudin-4 overexpression is associated with epigenetic derepression in gastric carcinoma. Lab Invest 91 (2011) 1652-1667.

[40] M.J. Kwon, S.S. Kim, Y.L. Choi, H.S. Jung, C. Balch, S.H. Kim, Y.S. Song, V.E. Marquez, K.P. Nephew, Y.K. Shin, Derepression of CLDN3 and CLDN4 during ovarian tumorigenesis is associated with loss of repressive histone modifications.

Carcinogenesis 31 (2010) 974-983.

[41] H. Fujita, Y. Hamazaki, Y. Noda, M. Oshima, N. Minato, Claudin-4 deficiency results in urothelial hyperplasia and lethal hydronephrosis. PLoS One 7 (2012) e52272.

[42] J.G. Smedley, 3rd, F.A. Uzal, B.A. McClane, Identification of a prepore large-complex stage in the mechanism of action of Clostridium perfringens enterotoxin. Infect Immun 75 (2007) 2381-2390.

[43] T. Kojima, D. Kyuno, N. Sawada, Targeting claudin-4 in human pancreatic cancer. Expert Opin Ther Targets 16 (2012) 881-887.

[44] S.M. Hashimi, S. Yu, N. Alqurashi, D.S. Ipe, M.Q. Wei, Immunotoxin-mediated targeting of claudin-4 inhibits the proliferation of cancer cells. Int J Oncol 42 (2013) 1911-1918.

[45] R. Saeki, M. Kondoh, H. Kakutani, K. Matsuhisa, A. Takahashi, H. Suzuki, Y. Kakamu, A. Watari, K. Yagi, A claudin-targeting molecule as an inhibitor of tumor metastasis. J Pharmacol Exp Ther 334 (2010) 576-582.

[46] X. Li, M. Iida, M. Tada, A. Watari, Y. Kawahigashi, Y. Kimura, T. Yamashita, A. Ishii-Watabe, T. Uno, M. Fukasawa, H. Kuniyasu, K. Yagi, M. Kondoh, Development of 
Kuwada et al.

an anti-claudin-3 and -4 bispecific monoclonal antibody for cancer diagnosis and therapy. J Pharmacol Exp Ther 351 (2014) 206-213.

[47] S. Edge, D.R. Byrd, C.C. Compton, A.G. Fritz, F.L. Greene, A. Trotti, AJCC Cancer Staging Manual 7th ed., Springer, New York, 2010. 
Kuwada et al.

Table 1. Association between CLDN4 expression and clinicopathological parameters in 86 cases of human bladder cancer

\begin{tabular}{|c|c|c|c|c|}
\hline \multirow[t]{2}{*}{ Parameters ${ }^{1)}$} & \multicolumn{3}{|c|}{ CLDN4 expression } & \multirow[t]{2}{*}{$P$-value } \\
\hline & Weak & Medium & High & \\
\hline Number & 19 & 47 & 20 & \\
\hline Age & $60(37-82)$ & $63(37-95)$ & $62(49-95)$ & $\mathrm{NS}^{2)}$ \\
\hline Sex (male:female) & $13: 6$ & $41: 6$ & $17: 3$ & NS \\
\hline \multicolumn{5}{|l|}{ Local invasion (pT) } \\
\hline pTa/pTis-pT1 & 7 & 17 & 1 & \\
\hline pT2 & 6 & 17 & 4 & \\
\hline pT3a & 3 & 5 & 5 & \\
\hline pT3b & 2 & 8 & 8 & \\
\hline pT4 & 0 & 0 & 2 & 0.0123 \\
\hline
\end{tabular}

Nodal metastasis $(\mathrm{pN})$

N0

19

47

17

N1

0

0

3

0.0059

Distant metastasis (pM)

M0

19

47

18

M1

0

0

2

0.0341 
Kuwada et al.

$\begin{array}{lcccc}\text { Stage } & & & & \\ \text { I } & 7 & 17 & 1 & \\ \text { II } & 11 & 22 & 9 & \\ \text { III } & 1 & 8 & 6 & 0.0011 \\ \text { IV } & 0 & 0 & 4 & \end{array}$

Grade

\begin{tabular}{lllll} 
Low & 11 & 22 & 8 & \\
High & 8 & 25 & 12 & NS \\
\hline
\end{tabular}

TNM classification criteria were used for $\mathrm{pT}, \mathrm{pN}, \mathrm{pM}$, stage, and grade [47]; NS, not significant 
Kuwada et al.

\section{Figure legends}

\section{Figure 1. CLDN specificity analysis}

4D3 binding to HT1080 cells expressing hCLDN1, 2, 3, 4, 5, 6, 7, 8, and 9, and mCLDN4-expressing $\mathrm{L}$ cells were examined by using FACSCalibur. Only hCLDN4-expressing HT1080 cells showed a high affinity for 4D3. (B) Immunoprecipitation by 4D3. CLDN4 was detected in 4D3-precipitant by 4D3 detection. CLDN1,3,7 were not detected in 4D3-precipitant. (C) Expressions of hCLDN1, 2, 3, 4, 5, 6, 7, 8, and 9 were examined by western blotting in parent HT1080 cells and CLDN4-transfected HT1080 cells treated with control or CLDN4 siRNAs. siRNA; short interference RNA, Ctr; control mixed siRNA, CL-4; CLDN4 siRNA, IP; immunoprecipitation, HC; heavy chain, LC; light chain.

Figure 2. Immunohistochemical detection of CLDN4 by 4D3 in bladder mucosa and urothelial carcinomas

(A) Non-cancerous urothelium. (B) Non-invasive urothelial carcinomas (UC); low grade, pTa, pN0. (C) Invasive UC; high grade, pT3, pN0. (D) Metastasizing UC, high grade, pT4, pN1, pM1. (E) A negative control using unimmunized rat IgG as a primary antibody. Bar, $100 \mu \mathrm{m}$.

\section{Figure 3. Effect of 4D3 on bladder cancer cells}

(A) CLDN4 mRNA expression and CLDN4 protein production in T24 (high-grade) and RT4 (low-grade) urothelial carcinoma cells. ß-actin and tubulin were used as loading controls. (B) Cell growth, (C) in vitro invasion, and (D) apoptosis analyses. (E-H) Multiple cell-layer microcarrier assay. (E) Epithelial growth factor (EGF) concentration in intra-carrier medium (CM, extra-carrier culture medium). (F) Expression and phosphorylation level of EGFR and extracellular signal-regulated kinase $1 / 2 \quad($ ERK1/2) by immunoblot analysis of the carrier-surface cells. (G) Vascular endothelial growth factor (VEGF) concentration in intra-carirrer medium. (H) mRNA expression of $V E G F$ and $H I F-1 \alpha$ (error bar, standard 
Kuwada et al.

deviation calculated from three independent experiments).

\section{Figure 4. Effect of concurrent treatment with $4 \mathrm{D} 3$ and cisplatin}

(A-B) Effect of concurrent treatment with cisplatin (CDDP) and 4D3 on T24 and RT4 cells. (C-D) Intracellular platinum concentrations with or without 4D3 treatment in T24 and RT4 cells. (E) A multiple cell layer permeability assay using peroxidase-conjugated IgG with various concentrations of 4D3 or control IgG in T24 cells. (F) A monolayer permeability assay using fluorescein isothianate (FITC)-dextran with 4D3 $(1 \mu \mathrm{g} / \mathrm{mL})$. Cytochalasin B $(\mathrm{CCB}, 1 \mu \mathrm{g} / \mathrm{mL}$ ) was used as a positive control [26]. (error bar, standard deviation calculated from three independent experiments; $\left.{ }^{*} P<0.01, \# P<0.0001\right)$.

\section{Figure 5. Effect of concurrent treatment with 4D3 and cisplatin in a mouse model}

(A) Treatment of subcutaneous T24 cell tumors with cisplatin (CDDP) and/or 4D3 (yellow arrow, administration). (B) Treatment of a lung T24-cell metastasis model with CDDP and/or 4D3. (C, D) Fluorescent examination of T24 subcutaneous tumor. (C) ICG-labeled 4D3 was detected by whole mouse fluorescence imaging. (D) Frozen section of the tumor was examined at $6 \mathrm{hrs}$ after administration of FITC-labeled 4D3 into the mouse peritoneal cavity.

(E) Effect of 4D3 $(1 \mathrm{mg} / \mathrm{kg})$ on permeability of T24 subcutaneous tumor examined by using a FITC-dextran bolus injection (bar, $50 \mu \mathrm{m}$ ). (F) FITC-positive area of subcutaneous tumors treated with or without 4D3 (1 mg/kg). (G) Survival of mice with T24 subcutaneous tumors (error bar, standard deviation calculated from 5 mice; $* P<0.01, * * P<0.001, \# P<0.0001$ ). 

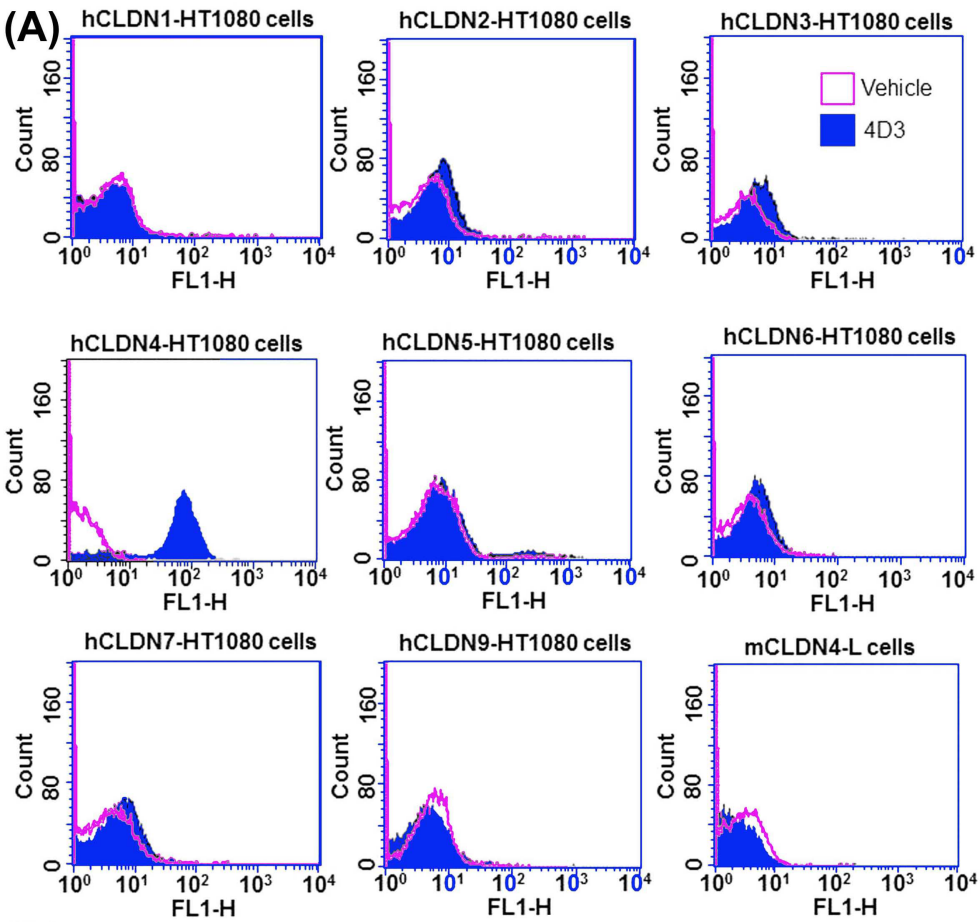

(B) SiRNA Ctr CL4 Ctr Crt Ctr Ctr Detect CL4 CL4 CL4 CL1 CL3 CL7

HC-

(C) CLDN

HT1080

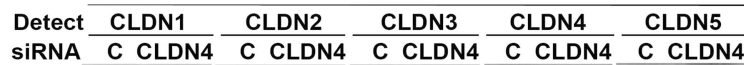

$\beta$-actin

\begin{tabular}{llllll} 
& \multicolumn{2}{c}{ HT1080 } & \multicolumn{2}{c}{$\begin{array}{c}\text { hCLDN4 } \\
\text {-HT1080 }\end{array}$} \\
\cline { 2 - 4 } Detect & CLDN6 & CLDN7 & CLDN8 & CLDN9 & CLDN4 \\
${$\cline { 3 - 3 }$} }$ & C CLDN4 & C CLDN4 & C CLDN4 & C CLDN4 & C CLDN4
\end{tabular}

CLDN24-

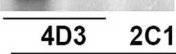
CLDN $\beta$-actin Coomassie blue IP 


\section{B}

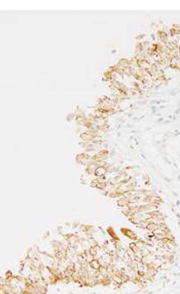

\section{Urothelium}

\section{G2, PTaNO}

C

\section{D}

E

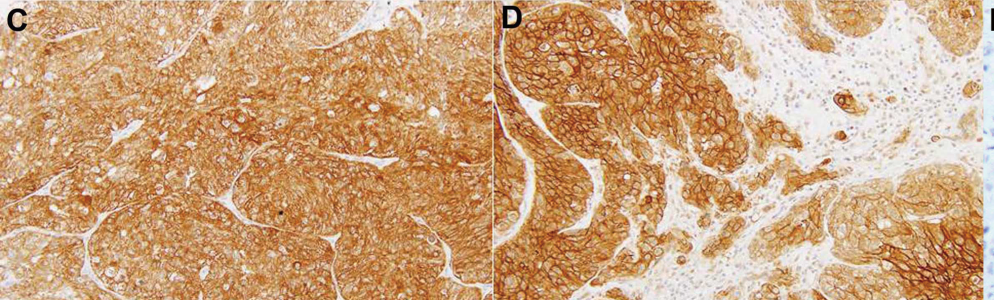

G3, pT3NO $\quad$ G3, pT4N1

Figure 2

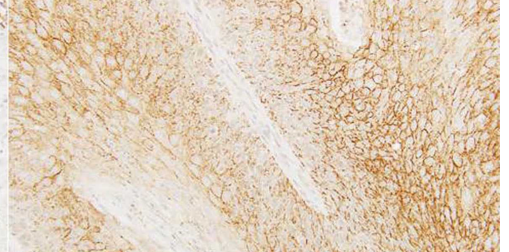


Figure 4
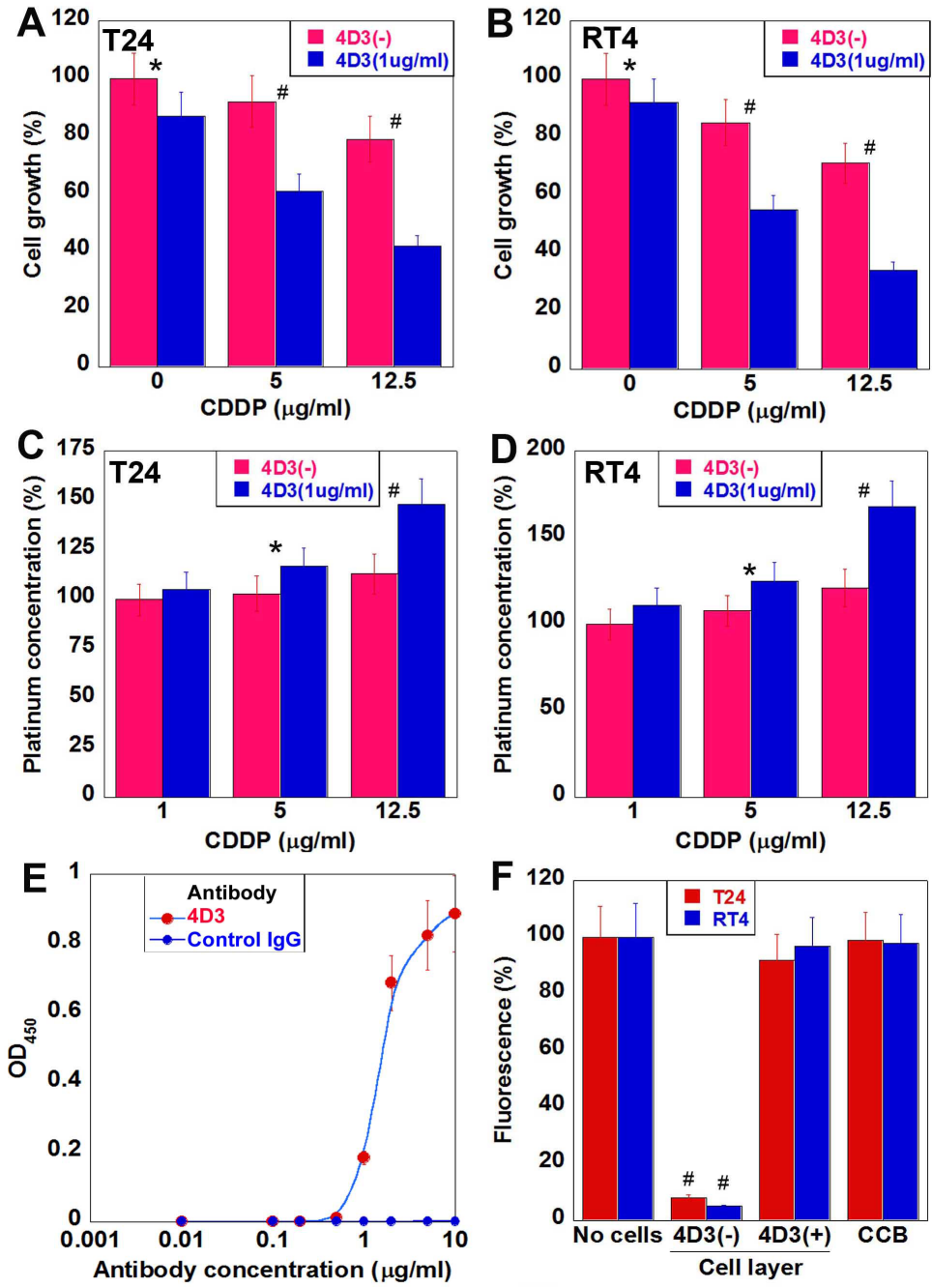


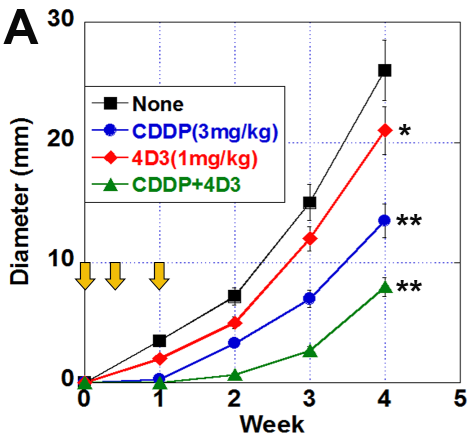

B $3 \quad$ Figure 5
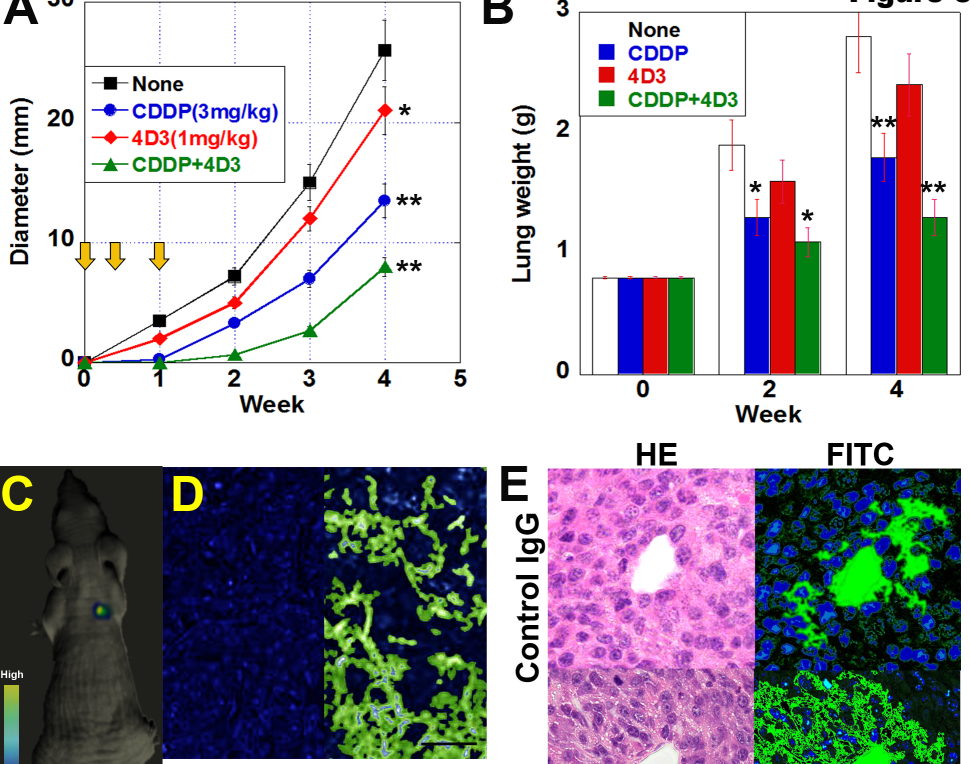

HE

FITC

Control IgG 4D3

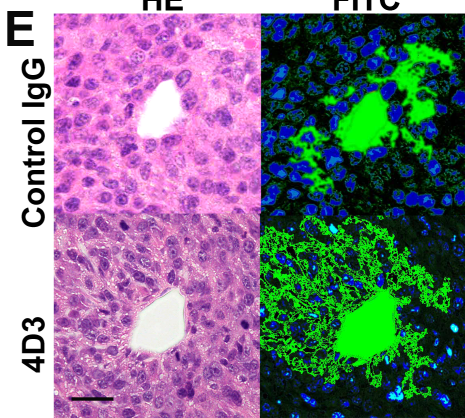

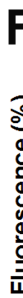
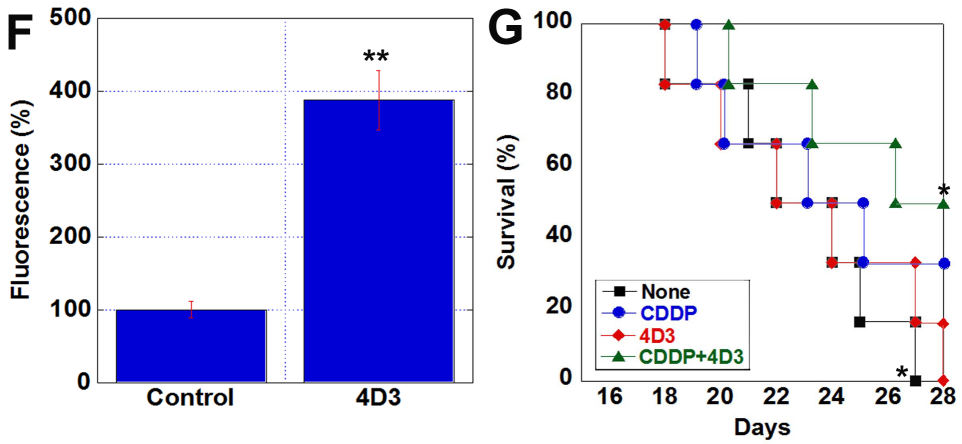